JOURNAL OF SYNCHROTRON RADIATION

ISSN 1600-5775

Received 16 March 2021

Accepted 23 October 2021

Edited by A. Stevenson, Australian Synchrotron, Australia

Keywords: longitudinal emittance; phase space profile; storage ring.

\section{Single-shot measurement of longitudinal phase space beam profile in an electron storage ring}

\author{
J. Kim, ${ }^{\text {a }}$ G. S. Jang, ${ }^{\mathrm{b}}$ B. H. Oh, ${ }^{\mathrm{c}}$ J. Lee ${ }^{\mathrm{c}}$ and S. Shin ${ }^{\mathrm{c} *}$

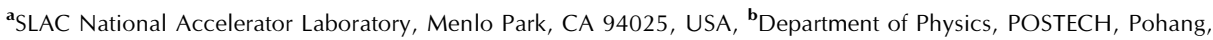 \\ Gyungbuk 37673, Republic of Korea, and ' Pohang Accelerator Laboratory, POSTECH, Pohang, Gyungbuk 37673 , \\ Republic of Korea. ${ }^{*}$ Correspondence e-mail: tlssh@postech.ac.kr
}

A novel scheme to measure the longitudinal emittance and phase space profile in an electron storage ring by using correlations between time and the vertical coordinate, and between energy and the horizontal coordinate, is proposed. This longitudinal profile measurement scheme will help to demonstrate recent results of advanced studies for manipulating the longitudinal beam profile and for investigating beam instability in an electron storage ring.

\section{Introduction}

Transverse bunch crabbing using a two-frequency crab cavity scheme (Zholents, 2015; Huang et al., 2019) provides the optimal solution to produce short-pulse (1-10 ps full width at half-maximum) X-rays in a storage ring. This scheme would enable increased precision of timing-mode studies of a large number of dynamic processes in materials as they function. A crab cavity can also be used as an injection kicker in a new onaxis injection scheme (Kim et al., 2019) that uses a transverse deflecting radio-frequency (RF) cavity to kick the incoming beam into an already populated bucket but with a timing offset from the synchronous phase.

A crab cavity can also be used to measure bunch length (Loew \& Altenmueller, 1965; Emma et al., 2000). A crab cavity couples the $y-z$ or $x-z$ planes, so bunch length is projected in the $y$ or $x$ dimension, respectively. The resolution of this bunch length measurement is limited by transverse emittances $\varepsilon_{x}$ and $\varepsilon_{y}$ unless a specialized beam transport line such as a chicane (Xiang \& Ding, 2010) is used. Regardless of using a crab cavity, the distribution of the energy deviation $\delta=$ $d p / p$ of a bunch can be projected in the $x$ dimension at which horizontal dispersion $\eta_{x}$ is large. The resolution of this is limited by the ratio $\varepsilon_{x} / \eta_{x}$. Combining these two principles enables measurement of the longitudinal profiles $(z-\delta)$ of a bunch in the $x-y$ plane.

A fourth-generation storage ring (4GSR) is an accelerator that provides small emittance so that the measurement can be made with high resolution. A 4GSR adopts a multi-bend achromat (MBA), that effectively suppresses natural emittance. The MAX IV 4GSR is currently operating; others, including APS-U, SPring-8-II, SLS-II, ALS-U, SIRIUS, ESRF-EBS and Korea-4GSR, are being designed, constructed or commissioned [Streun, 2017; Steier et al., 2016; Liu et al., 2013; see also design reports for MAX IV (https://www. maxiv.lu.se/acceleratorsbeamlines/accelerators/accelerator documentation/max-iv-ddr), APS Upgrade (https:/www. aps.anl.gov/APSUpgrade), SPring-8-II (http://rsc.riken.jp/ 
eng/index.html) and ESRF-EBS (https://indico.psi.ch/event/ 5589/)]. All of these rings use the MBA concept. A single cell of an MBA lattice has M-1 dispersion maxima with similar amplitudes, and one of these maxima can be used as a watching point for the measurement using a crab cavity. A hybrid MBA lattice such as APS-U, ESRF-EBS or Korea4GSR provides two large dispersion bumps at the edge of a cell to enable effective correction of chromaticity, and the region of the large dispersion bumps is a desirable choice as a watching point.

In this study, we show that in a storage ring that uses a hybrid MBA lattice the measurement on longitudinal bunch profiles gives good resolution without the need for other additional magnets. In Section 2, we recall the matrix formalism between two arbitrary points of a storage ring, and projection of longitudinal $(z-\delta)$ beam profiles on the transverse $(x-y)$ plane by using a crab cavity. In Section 3, we briefly introduce PAL-4GSR, which is used as an example lattice for the novel measurement scheme. We also calculated the intrinsic resolution of the measurement in PAL-4GSR. In Section 4, we present a numerical simulation of the measurement of the longitudinal profile by using the PAL4GSR lattice. We also examine the resolution of the measurement when wakefield data are included.

\section{Coupling of the $y-z$ plane}

Projection of the $z-\delta$ beam profile into the $x-y$ plane can be described by linear optics theory. A thin crab cavity of TM110 mode has a linearized function as follows (Huang, 2016),

$$
T_{\mathrm{c}}=\left(\begin{array}{cccccc}
1 & 0 & 0 & 0 & 0 & 0 \\
0 & 1 & 0 & 0 & 0 & 0 \\
0 & 0 & 1 & 0 & 0 & 0 \\
0 & 0 & 0 & 1 & \epsilon & 0 \\
0 & 0 & 0 & 0 & 1 & 0 \\
0 & 0 & \epsilon & 0 & 0 & 1
\end{array}\right)
$$

where $\epsilon=e V k / E_{0}$, in which $e$ is the electron charge, $V[\mathrm{~V}]$ is the maximum voltage of a crab cavity, $k$ is the angular wavenumber and $E_{0}[\mathrm{eV}]$ is the nominal energy of a beam. In a storage ring, a linear matrix from arbitrary position 1 to arbitrary position 2 is given as (Chao, 2002)

$$
M_{12}=\left(\begin{array}{cccccc}
R_{11} & R_{12} & 0 & 0 & 0 & \eta_{2}-R_{11} \eta_{1}-R_{12} \eta_{1}^{\prime} \\
R_{21} & R_{22} & 0 & 0 & 0 & \eta_{2}^{\prime}-R_{21} \eta_{1}-R_{22} \eta_{1}^{\prime} \\
0 & 0 & R_{33} & R_{34} & 0 & 0 \\
0 & 0 & R_{43} & R_{44} & 0 & 0 \\
A & B & 0 & 0 & 1 & C-A \eta_{1}-B \eta_{1}^{\prime} \\
0 & 0 & 0 & 0 & 0 & 1
\end{array}\right) .
$$

Definitions of each matrix element can be found in Appendix $A$. When initial beam coordinates at the position 1 are given as $X_{1}=\left(x_{1}, x_{1}^{\prime}, y_{1}, y_{1}^{\prime}, z_{1}, \delta_{1}\right)$, its continuous mapping via $T_{\mathrm{c}}$ and $M_{12}$ becomes

$$
X_{2}=M_{12} T_{\mathrm{c}} X_{1}=\left(\begin{array}{c}
R_{11} x_{1}+R_{12} x_{1}^{\prime} \\
+\left(\eta_{2}-R_{11} \eta_{1}-R_{12} \eta_{1}^{\prime}\right) y_{1} \epsilon \\
+\left(\eta_{2}-R_{11} \eta_{1}-R_{12} \eta_{1}^{\prime}\right) \delta_{1} \\
R_{21} x_{1}+R_{22} x_{1}^{\prime} \\
+\left(\eta_{2}^{\prime}-R_{21} \eta_{1}-R_{22} \eta_{1}^{\prime}\right) y_{1} \epsilon \\
+\left(\eta_{2}^{\prime}-R_{21} \eta_{1}-R_{22} \eta_{1}^{\prime}\right) \delta_{1} \\
R_{33} y_{1}+R_{34} y_{1}^{\prime}+R_{34} z_{1} \epsilon \\
R_{43} y_{1}+R_{44} y_{1}^{\prime}+R_{44} z_{1} \epsilon \\
A x_{1}+B x_{1}^{\prime}+\left(C-A \eta_{1}-B \eta_{1}^{\prime}\right) y_{1} \epsilon \\
+\left(C-A \eta_{1}-B \eta_{1}^{\prime}\right) \delta_{1} \\
y_{1} \epsilon+\delta_{1}
\end{array}\right) .
$$

Let us assume that position 1 is located at one of the achromats of a storage ring $\left(\eta_{1}=0\right)$, and position 2 is at a point that has large dispersion. If $\psi_{x} \simeq(2 n+1) \pi$ and $\psi_{y} \simeq$ $[(2 n+1) / 2] \pi$, contributions from $R_{12}$ and $R_{33}$ are negligible. The magnitude of $R_{11}$ is approximately $\left(\beta_{x 2} / \beta_{x 1}\right)^{1 / 2}$ when $\psi_{x} \simeq$ $(2 n+1) \pi$, and it is conservatively less than 3 as the ratio of $\beta_{x 2}$ over $\beta_{x 1}$ is less than 10 for most lattices. Specifically, when emittance $\simeq 100 \mathrm{pm}, \delta_{1} \simeq 0.001$ and $\eta_{2} \simeq 0.10 \mathrm{~m}$, the contribution from $\eta_{2} \sigma_{\delta_{1}}$ at $x_{2}$ is one order larger than that from $\sigma_{x_{1}}$ (i.e. $\sigma_{x_{1}} \simeq 10^{-5} \mathrm{~m}$ and $\eta_{2} \sigma_{\delta_{1}} \simeq 10^{-4}$ ), which means we can expect projection between $x_{2}$ and $\eta_{2} \sigma_{\delta_{1}}$. Likewise, $y_{2}$ is dominated by $R_{34} z_{1} \epsilon$ as $\sigma_{y_{1}^{\prime}}$ is of the order of $10^{-6}$ but $z_{1}$ is of the order of $10^{-3}$ and $\epsilon$ has order $10^{-2}$. Clearly, smaller emittance will lead to better resolution.

Hence, $x$ and $y$ coordinates at position 2 are expressed as

$$
\left(\begin{array}{c}
x_{2} \\
y_{2}
\end{array}\right) \cong\left(\begin{array}{cc}
0 & \eta_{2} \\
R_{34} \epsilon & 0
\end{array}\right)\left(\begin{array}{c}
z_{1} \\
\delta_{1}
\end{array}\right)
$$

which shows the projection of the $z-\delta$ plane onto the $x-y$ plane. Its resolution is mainly dependent on whether the contributions from $x_{1}, x_{1}^{\prime}, y_{1}$ and $y_{1}^{\prime}$ are negligible and whether $\psi_{x} \simeq(2 n+1) \pi$ and $\psi_{y} \simeq[(2 n+1) / 2] \pi$. If they are, then $R_{34}$ has $\sin \left(\psi_{y}\right)$ dependence, so large $\eta_{2}$ leads to increased magnification for the $x$ plane, and $\psi_{y}$ close to $[(2 n+1) / 2] \pi$ leads to increased magnification for the $y$ plane.

A hybrid MBA lattice such as ESRF-EBS, APS-U, PAL4GSR satisfies the above-described conditions. Due to the common $\left(\psi_{x}, \psi_{y}\right)=(3 \pi, \pi)$ phase advance between two dispersion bumps in a cell of a hybrid MBA lattice, each cell has a similar phase advance. Specifically, a hybrid MBA lattice has $\varphi_{x} \simeq 0.9 \pi$ and $\varphi_{y} \simeq 0.4 \pi$ from the center of the long straight section to the nearest dispersion bump. Hence, choosing the position of a crab cavity satisfying $\varphi_{y}=\pi / 2$ is accompanied by $\varphi_{x} \simeq \pi$.

\section{PAL-4GSR and the intrinsic resolution}

The PAL-4GSR storage ring is a hybrid seven-bend achromat (H7BA) lattice with a horizontal emittance of $90 \mathrm{pm}$. The 
Table 1

Parameters relevant to the PAL-4GSR lattice.

\begin{tabular}{lll}
\hline Parameter & Value & Unit \\
\hline Energy & 3 & $\mathrm{GeV}$ \\
Emittance (flat / round) & $90 / 58$ & $\mathrm{pm}$ \\
Circumference & 569.96 & $\mathrm{~m}$ \\
Tune $(x / y)$ & $47.545 / 18.203$ & - \\
Natural chromaticity $(x / y)$ & $-96.0 /-57.9$ & - \\
Radiation loss per turn & $0.468($ without insertion device) & $\mathrm{MeV}$ \\
Momentum compaction & $1.45 \times 10^{-4}$ & \\
Damping partition $(\mathrm{H} / \mathrm{V} / \mathrm{L})$ & $1.82 / 1.0 / 1.18$ & $\mathrm{~ms}$ \\
Damping time $(\mathrm{H} / \mathrm{V} / \mathrm{L})$ & $13.37 / 24.35 / 20.66$ & $\mathrm{MV}$ \\
Main RF voltage & 2.15 &
\end{tabular}

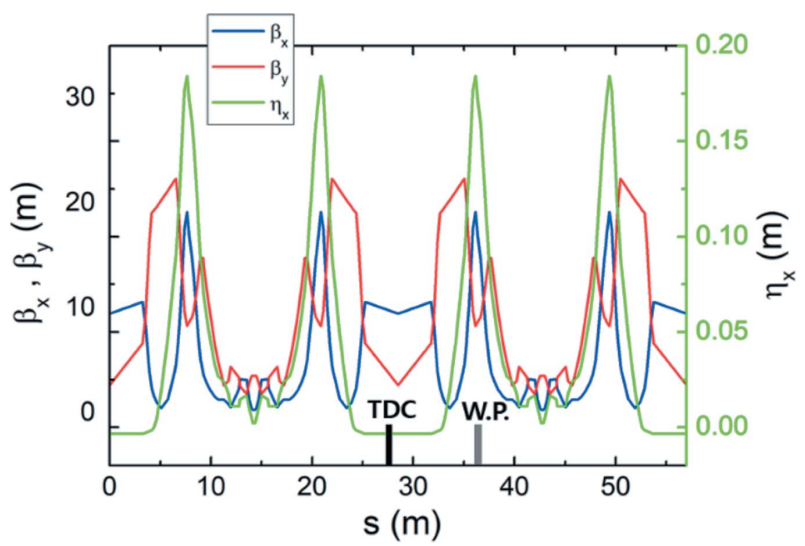

Figure 1

Twiss functions of PAL-4GSR (two cells) and the position of the transverse deflecting cavity and watching point.

ring has a circumference of $570 \mathrm{~m}$, and is composed of 20 symmetrical cells. From experience on PLS-II (Shin et al., 2013), the length of the straight section is considered to be $6.5 \mathrm{~m}$ to accommodate two SCRF modules in one straight section. The PAL-4GSR lattice (Table 1, Fig. 1) contains a $2 \mathrm{~T}$ super-bend in the central dipole to produce radiation with a critical energy of $12 \mathrm{keV}$.

The concepts of the ESRF-EBS and APS-U lattices were adopted in the PAL-4GSR lattice. The dispersion was deliberately enlarged between the first and second dipoles and between the sixth and seventh dipoles, then three chromatic sextupoles were located in this dispersion bump region to

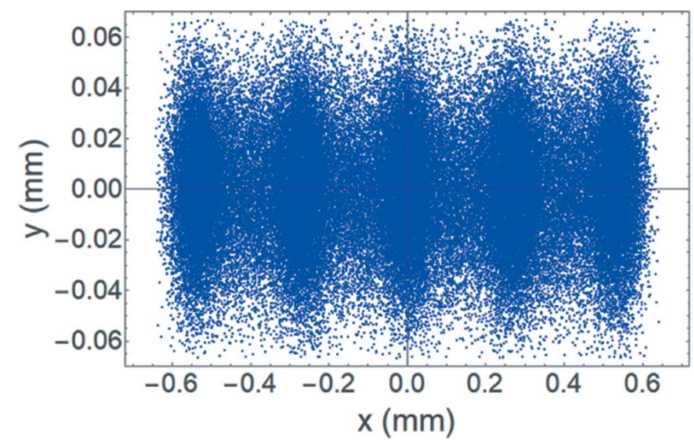

(a)



Figure 2

Longitudinal profiles of 100000 particles at the position of the crab cavity for examination of intrinsic resolution. Particles are distributed with a delta function in longitudinal phase space.

reduce the strength required to control the chromaticity. The betatron phase advances between the two dispersion bumps were set to $\Delta \varphi_{x} \simeq 3 \pi$ in the horizontal plane and $\Delta \varphi_{y} \simeq \pi$ in the vertical plane; as a result, nonchromatic effects of the sextupoles are canceled out naturally. To minimize natural emittance, five-step longitudinal gradient dipoles and reverse bending magnets were considered (Streun \& Wrulich, 2015; Nagaoka \& Wrulich, 2007; Delahaye \& Potier, 1989; Streun, 2014).

We performed tracking simulation to examine the resolution of the measurement using a transverse deflecting cavity (TDC) on PAL-4GSR. For tracking simulation, we used elegant software (Borland, 2000). A total of 100000 particles were generated at the position of the TDC (Fig. 1). They have a $58 \mathrm{pm}$ (standard deviation) Gaussian distribution with matched Twiss functions in the horizontal and vertical planes, and ten lines of uniform density with maximum values of $\pm 0.3 \%$ and $\pm 24 \mathrm{~mm}$ in the longitudinal plane (Fig. 2). A watching point located at the left dispersion bump has a vertical phase advance of $\pi / 2$ from the position of the TDC. For a TDC, we set a voltage of $4.5 \mathrm{MV}$ and a frequency of $750 \mathrm{MHz}$, which yield $\epsilon=0.02358$. When the TDC was off, we observed five resolved distributions on the $x$ axis at the watching point due to the correlation $x_{2} \simeq \eta_{2} \delta_{1}$ [Figs. 3(a) and $4(a)$ ]. In the $y$ axis, the distribution was not resolved [Figs. 3(a) and 4(b)]. Standard deviations of Gaussian peaks were

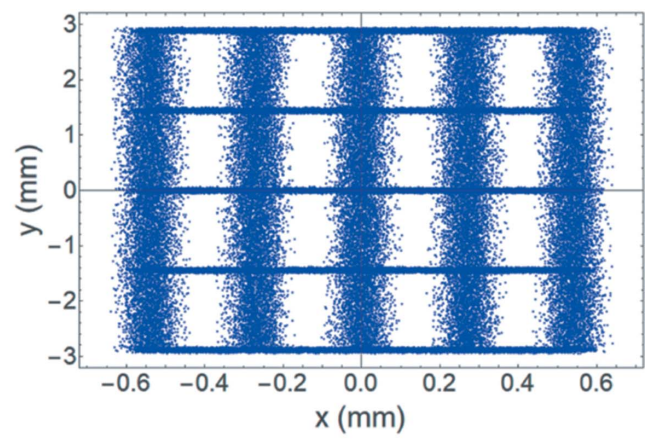

(b)

Figure 3

Beam distribution at the watching point with 100000 particles prepared for examination of intrinsic resolution. (a) TDC off; (b) TDC on. 
$0.037 \mathrm{~mm}$ on the $x$ axis and $0.022 \mathrm{~mm}$ on the $y$ axis, which are explained by $\sigma_{x_{2}} \simeq R_{11} \sigma_{x_{1}}+R_{12} \sigma_{x_{1}^{\prime}}$ and $\sigma_{y_{2}} \simeq R_{33} \sigma_{y_{1}}+R_{34} \sigma_{y_{1}^{\prime}}$. When the TDC was on, the $x$ axis was not affected, but five resolved distributions appeared on the $y$ axis [Fig. 3(b)]. The effective length on the $y$ axis increased from $0.13 \mathrm{~mm}$ to $5.95 \mathrm{~mm}$ due to $y_{2} \simeq R_{34} \epsilon z_{1}$ whereas the standard deviations of the Gaussian peaks did not change. These processes allow clear resolution of the five Gaussian peaks [Fig. 4(c)]. From the revealed Gaussian peaks with the use of a line distribution on the longitudinal plane, we define the intrinsic resolution on an axis as $R_{\mathrm{I}}=$ (distance between adjacent Gaussian peak) / (standard deviation of a Gaussian peak). $R_{\mathrm{I}}$ increases with increase in the clarity of distinction of the Gaussian peak on an axis. PAL-4GSR has $R_{\mathrm{I}}=6.69$ on the $x$ axis and $R_{\mathrm{I}}=64.68$ on the $y$ axis. The dispersion bump (for $\delta-x$ correlation) increases and the voltage of the TDC increases (for $z-y$ correlation), so the resolution can be increased. Adjustment of dispersion bumps is constrained by lattice requirements, but the voltage of the TDC can be increased further without affecting lattice requirements and we can expect much better resolution than is currently achieved.

\section{Application on PAL-4GSR}

A Gaussian bunch of 100000 particles in an equilibrium state was generated for the six-dimensional phase space with the round-beam mode. It had $58 \mathrm{pm}$ emittance in both horizontal and vertical phase spaces. It should be mentioned that, for simplicity, distributed coupling errors are considered along the ring except for the region from the TDC and watching point to generate round beam. With a main RF voltage of $2.15 \mathrm{MV}$, its longitudinal distribution had $\sigma_{\delta}=0.00108$ and $\sigma_{z}=7.677 \mathrm{~mm}$ (Table 2). For a TDC, a voltage of $4.5 \mathrm{MV}$ and a frequency of $750 \mathrm{MHz}$ were also used.

\subsection{Longitudinal emittance measurement}

The longitudinal emittance can be written with the standard deviation as

$$
\varepsilon_{z}=\sigma_{z} \sigma_{\delta}
$$

Here, using equation (4), the standard deviations $\sigma_{z}$ and $\sigma_{\delta}$ can be approximately converted to
Table 2

Particle distribution at the equilibrium.

\begin{tabular}{lll}
\hline Parameter & Value & Unit \\
\hline Horizontal emittance & 58 & $\mathrm{pm}$ \\
Vertical emittance & 58 & $\mathrm{pm}$ \\
Energy spread (r.m.s.) & 0.00108 & - \\
Bunch length (r.m.s.) & 7.677 & $\mathrm{~mm}$ \\
\hline
\end{tabular}

$$
\sigma_{x} \simeq \eta_{2} \sigma_{\delta}
$$

and

$$
\sigma_{y} \simeq R_{34} \epsilon \sigma_{z}
$$

respectively. Therefore, measurements of $\sigma_{x}$ and $\sigma_{y}$ directly lead to estimates of longitudinal emittance. The generated Gaussian bunch is deflected at the TDC. After a $\pi / 2$ phase advance (at a watching point), the bunch's projection on the $x-y$ plane has a clear Gaussian distribution [Fig. 5(b)], with $\sigma_{x}=0.197 \mathrm{~mm}$ and $\sigma_{y}=0.922 \mathrm{~mm}$. From these values, reprojection using equation (4) gives $\sigma_{\delta}=1.096 \times 10^{-3}$ and $\sigma_{z}=$ $7.680 \mathrm{~mm}$, which have $1.46 \%$ and $0.04 \%$, respectively, error relative to the original values. As a result, the longitudinal emittance from the measurement is calculated to be $\varepsilon_{z}=$ $8.417 \times 10^{-6} \mathrm{~m}$, which is a $1.52 \%$ error relative to the original value. The original longitudinal profile at the TDC and the profile (Fig. 6) were obtained from re-projection using equation (4).

\subsection{Measurement of the longitudinal phase space profile with instability}

Beam manipulation in the longitudinal phase can be used to generate coherent radiation (Ratner \& Chao, 2010) and beam dynamics in the longitudinal phase space to enable exploration of a new injection scheme (Kim et al., 2019; Aiba et al., 2015; Jiang et al., 2016; Jiang \& Xu, 2018; Kuske et al., 2020). These goals invoke a need to measure the longitudinal beam profile. To generate a special shape in the longitudinal phase space, instabilities were deliberately induced by using wakefield data - here the impedance data of APS (Chae, 2003, 2007; Lindberg \& Blednykh, 2015). However, PAL-4GSR and APS have different characteristics such as circumference (PAL-



(a)

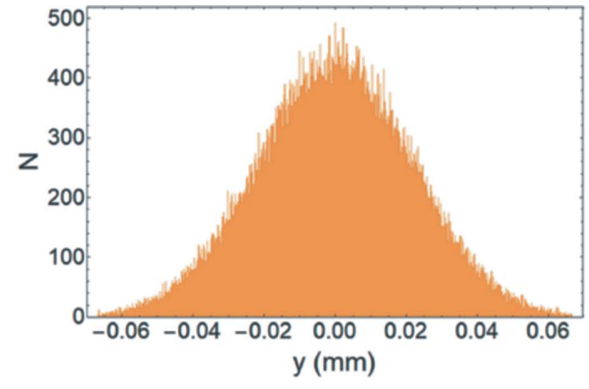

(b)

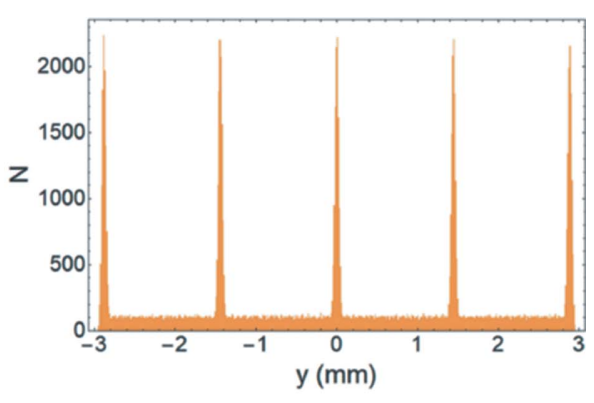

(c)

Figure 4

Density distribution on the $x$ and $y$ axis for the beam distribution shown in Fig. 3. (a) Density on the $x$ axis when the TDC is on or off, (b) density on the $y$ axis when the TDC is off, and (c) density on the $y$ axis when the TDC is on. 


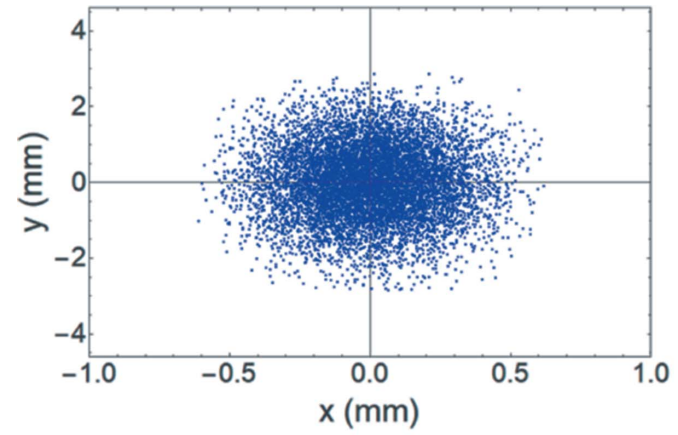

(a)

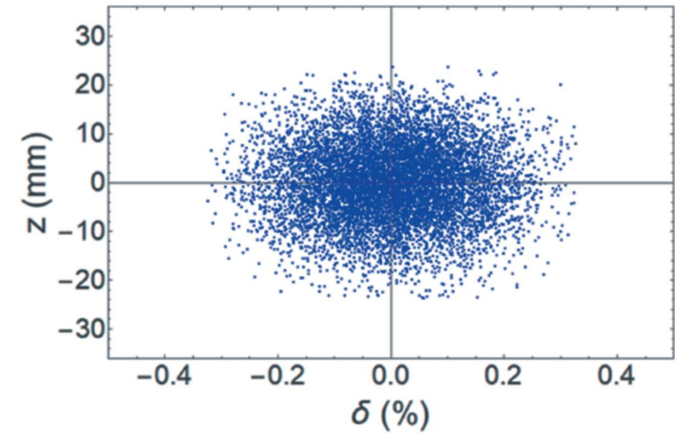

(b)

Figure 5

(a) $x-y$ projection at the watching point and $(b)$ longitudinal profile at the position of the crab cavity. No wakefield included.

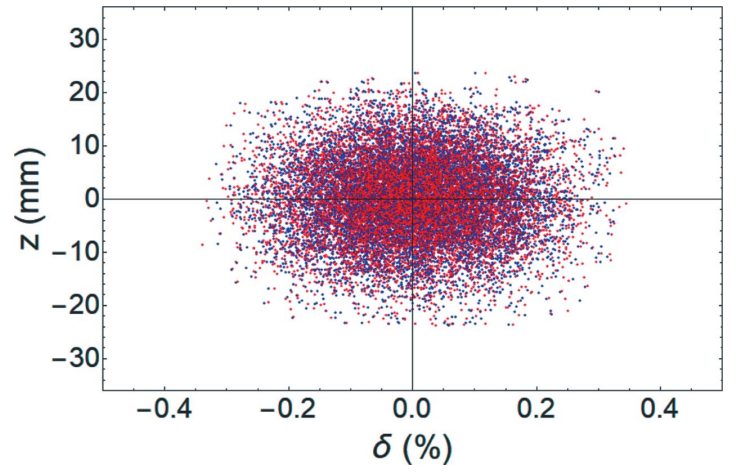

Figure 6

Comparison of the original longitudinal profile at the crab cavity position (blue dots) and re-projection from equation (5) (red dots). No wakefield included.

4GSR: $570 \mathrm{~m}$; APS: $1100 \mathrm{~m}$ ), nominal energy (PAL-4GSR: $3 \mathrm{GeV}$; APS: $7 \mathrm{GeV}$ ), so use of these data can invoke instabilities in PAL-4GSR. We first estimated the current threshold $I_{\text {threshold }}$ of the strong instability by following Boussard's criterion (Boussard, 1975),

$$
I_{\text {threshold }}=(2 \pi)^{3 / 2} \frac{\alpha_{\mathrm{c}} \sigma_{z} E \sigma_{\delta}^{2}}{c T_{0}\left|Z_{\|} / n\right|},
$$

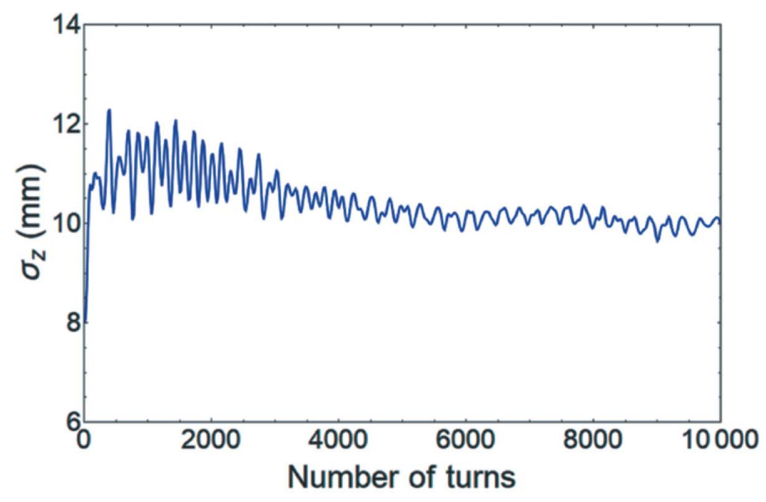

(a) where $\alpha_{\mathrm{c}}$ is the momentum compaction, $\sigma_{z}$ is the equilibrium bunch length, $\sigma_{\delta}$ is the equilibrium relative energy spread, $c$ is the speed of light, $T_{0}$ is the revolution time, and $\left|Z_{\|} / n\right|$ is the effective longitudinal impedance. This conservative estimate gives $I_{\text {threshold }} \simeq 0.37 \mathrm{~mA}$; therefore we used a single bunch current of $7.7 \mathrm{~mA}$ (or single bunch charge of $15 \mathrm{nC}$ ) which is sufficiently high above the estimated threshold. Including the impedance data, a tracking simulation was conducted using a Gaussian bunch of 100000 particles in the equilibrium state. Oscillations of r.m.s. bunch length and r.m.s. energy spread along a number of turns show that both quantities increased rapidly after tracking started, reached their maximum within 2000 turns and eventually converged to a new equilibrium at $\sim 10000$ turns (Fig. 7). The new equilibrium with the impedance data has 1.3 times larger bunch length and 1.8 times larger energy spread, compared with the original equilibrium. Those results imply that strong instability was driven well.

We next examined longitudinal beam profiles at 10000 turns (Fig. 8). After the kick of the TDC was applied, the longitudinal phase space was projected to the $x-y$ plane. The central beam distribution split up and the outer beam distribution seems to have a spiral shape. Also, the center of the bunch was moved to $\sim 0.3 \mathrm{~mm}$ on the $x$ axis. The overlap of the original longitudinal profile [Fig. $8(a)$ ] and re-projection from the $x-y$ plane [Fig. 8(b)] using equation (4) agreed well (Fig. 9).

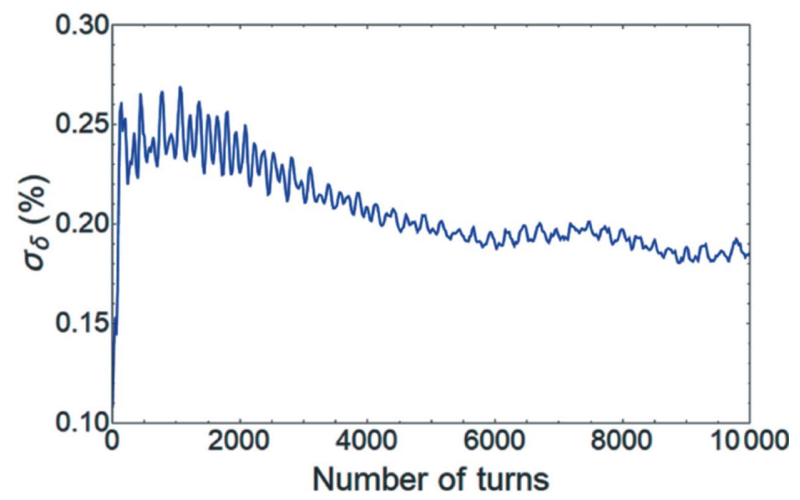

(b)

Figure 7

Oscillation of $(a)$ r.m.s. bunch length and $(b)$ r.m.s. energy spread versus turns when impedance data are included. 

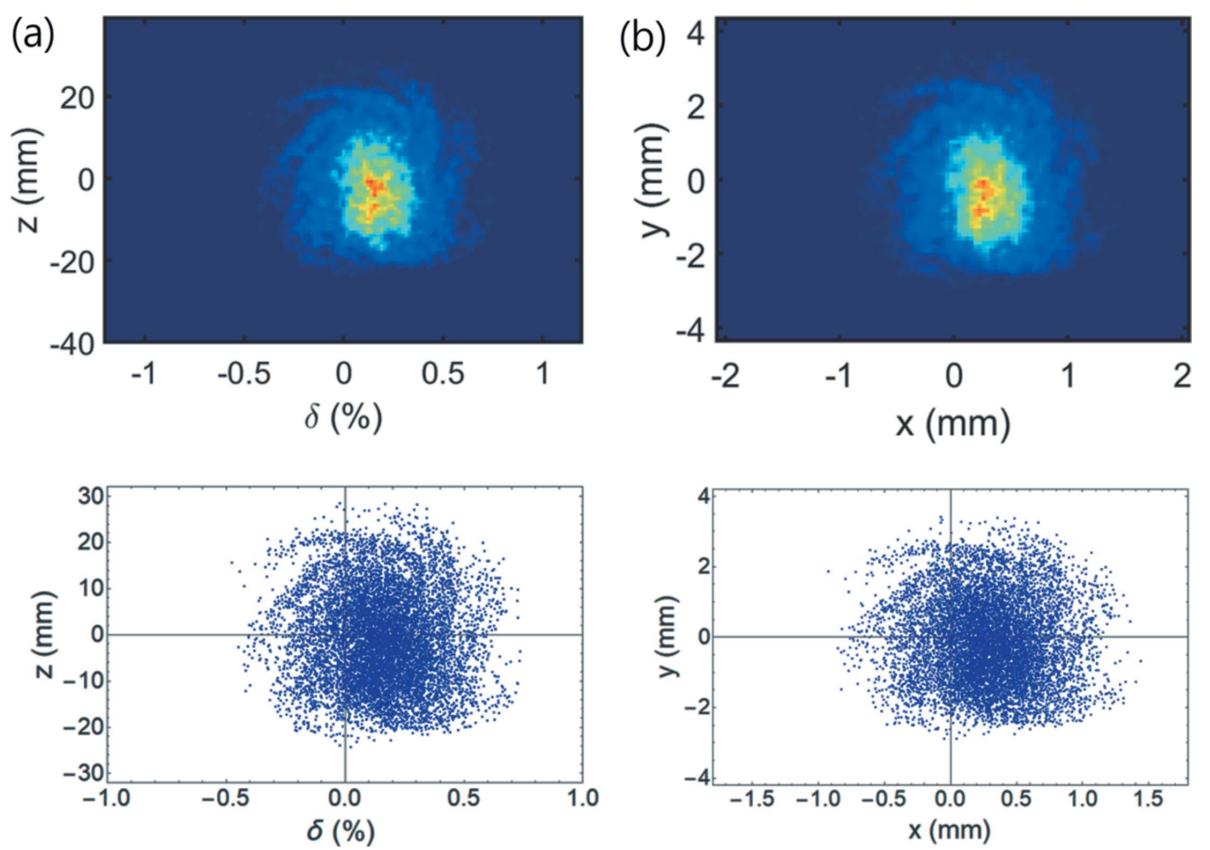

Figure 8

Longitudinal profiles at $(a)$ the position of the crab cavity, and $(b)$ the $x-y$ projection at the watching point. Wakefield is included.

\section{Conclusion}

We have described a novel scheme to measure the emittance and phase space profile in the longitudinal plane by using correlations between time and the vertical coordinate, and between energy and the horizontal coordinate. A large dispersion bump has a strong correlation with energy and the horizontal coordinate, and the crab cavity has a strong correlation with time and the vertical coordinate. As a result, longitudinal emittance was estimated with $<1.52 \%$ error in the PAL-4GSR lattice and micro-bunching instability was observed at the synchrotron radiation source point. This longitudinal profile measurement scheme will help to guide the manipulation of beams in longitudinal phase space.

\section{APPENDIX $A$}

Definitions of each matrix element of equation (2)

$$
\begin{gathered}
R_{11}=\left(\frac{\beta_{x 2}}{\beta_{x 1}}\right)^{1 / 2}\left(\cos \psi_{x}+\alpha_{x 1} \sin \psi_{x}\right), \\
R_{12}=\left(\beta_{x 1} \beta_{x 2}\right)^{1 / 2} \sin \psi_{x}, \\
R_{21}=-\frac{1}{\left(\beta_{x 1} \beta_{x 2}\right)^{1 / 2}}\left[\left(1+\alpha_{x 1} \alpha_{x 2}\right) \sin \psi_{x}-\left(\alpha_{x 1}-\alpha_{x 2}\right) \cos \psi_{x}\right], \\
R_{22}=\left(\frac{\beta_{x 1}}{\beta_{x 2}}\right)^{1 / 2}\left(\cos \psi_{x}-\alpha_{x 2} \sin \psi_{x}\right), \\
R_{33}=\left(\frac{\beta_{y 2}}{\beta_{y 1}}\right)^{1 / 2}\left(\cos \psi_{y}-\alpha_{y 1} \sin \psi_{y}\right),
\end{gathered}
$$

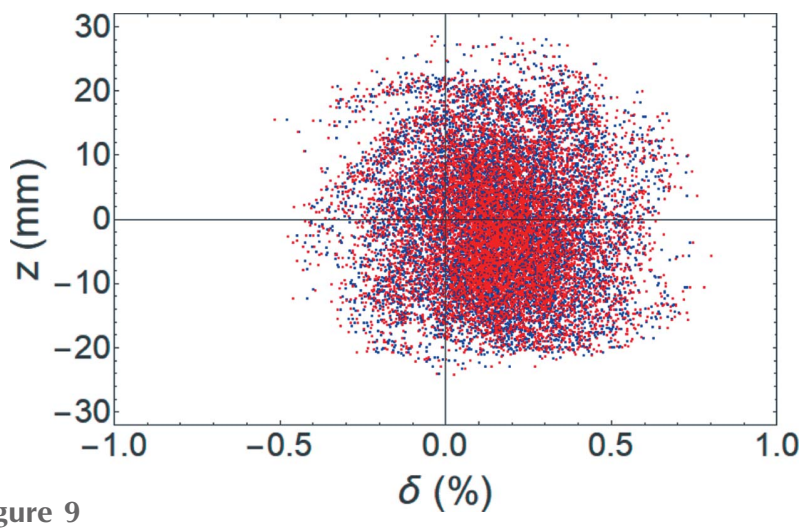

Comparison of the original longitudinal profile at the crab cavity position (blue dots) and re-projection of the $x-y$ profile at the monitor position using equation (4) (red dots). Wakefield is included for a single bunch of $15 \mathrm{nC}$.

$$
\begin{gathered}
R_{34}=\left(\beta_{y 1} \beta_{y 2}\right)^{1 / 2} \sin \psi_{y}, \\
R_{43}=-\frac{1}{\left(\beta_{y 1} \beta_{y 2}\right)^{1 / 2}}\left[\left(1+\alpha_{y 1} \alpha_{y 2}\right) \sin \psi_{y}-\left(\alpha_{y 1}-\alpha_{y 2}\right) \cos \psi_{y}\right] \\
R_{44}=\left(\frac{\beta_{y 1}}{\beta_{y 2}}\right)^{1 / 2}\left(\cos \psi_{y}-\alpha_{y 2} \sin \psi_{y}\right), \\
A=R_{21} \eta_{2}-R_{11} \eta_{1}^{\prime}+\eta_{1}^{\prime} \\
B=-R_{12} \eta_{2}^{\prime}+R_{22} \eta_{2}-\eta_{1}, \\
C=-\int_{s_{1}}^{s_{2}} \frac{\eta(s)}{\rho(s)} \mathrm{d} s .
\end{gathered}
$$


$\alpha, \beta$ are typical beta functions, $\eta, \eta^{\prime}$ are dispersion functions, with corresponding subscript. $\psi_{x}$ and $\psi_{y}$ are, respectively, the horizontal and vertical phase advances between positions 1 and 2 .

\section{Acknowledgements}

We thank G.-R. Hahn and T. Ha (PAL) for providing helpful information and many useful discussions.

\section{Funding information}

Funding for this research was provided by: National Research Foundation of Korea (grant No. NRF-2019R1C1C1003412; grant No. NRF-2019R1A2C1004862).

\section{References}

Aiba, M., Böge, M., Marcellini, F., Saá Hernández, A. \& Streun, A. (2015). Phys. Rev. ST Accel. Beams, 18, 020701.

Borland, M. (2000). Elegant: A flexible SDDS-compliant code for accelerator simulation, Report No. LS-287. Advanced Photon Source, Argonne, IL, USA.

Boussard, D. (1975). Technical Report LabII/RF/Int/75-2. CERN, Geneva, Switzerland.

Chae, Y. (2003). Proceedings of the 2003 Particle Accelerator Conference (PAC2003), 12-16 May 2003, Portland, OR, USA, p. 3017.

Chae, Y.-C. (2007). Proceedings of the 2007 Particle Accelerator Conference (PAC2007), 25-29 June 2007, Albuquerque, NM, USA, p. 4336.

Chao, A. (2002). Lecture notes on topics in accelerator physics. Technical Report SLAC-PUB-9574. SLAC National Accelerator Laboratory, Menlo Park, CA, USA.

Delahaye, J. P. \& Potier, R. (1989). Proceedings of the 1989 Particle Accelerator Conference (PAC1989), 20-23 March 1989, Chicago, IL, USA, pp. 1611-1613.

Emma, P., Frisch, J. \& Krejcik, P. (2000). A transverse RF deflecting structure for bunch length and phase space diagnostics. Technical Report LCLS-TN-00-12. SLAC National Accelerator Laboratory, Menlo Park, CA, USA.
Huang, X. (2016). Phys. Rev. Accel. Beams, 19, 024001.

Huang, X., Hettel, B., Rabedeau, T., Safranek, J., Sebek, J., Tian, K., Wootton, K. P. \& Zholents, A. (2019). Phys. Rev. Accel. Beams, 22, 090703.

Jiang, B. C., Zhao, Z. T., Tian, S. Q., Zhang, M. Z. \& Zhang, Q. L. (2016). Nucl. Instrum. Methods Phys. Res. A, 814, 1-5.

Jiang, S. \& Xu, G. (2018). Phys. Rev. ST Accel. Beams, 21, 110701.

Kim, J., Jang, G., Yoon, M., Oh, B., Lee, J., Ko, J., Parc, Y., Ha, T., Kim, D., Kim, S. \& Shin, S. (2019). Phys. Rev. Accel. Beams, 22, 011601.

Kuske, P., Li, J. \& Aiba, M. (2020). Phys. Rev. Accel. Beams, 23, 030701.

Lindberg, R. R. \& Blednykh, A. (2015). Proceedings of the Sixth International Particle Accelerator Conference (IPAC2015), 3-8 May 2015, Richmond, VA, USA, pp. 1822-1824. TUPJE077.

Liu, L. N., Milas, A. H. C., Mukai, X. R., Resende, A. R. D. \& Rodriguet Sa, F. H. (2013). Proceedings of the 4th International Particle Accelerator Conference (IPAC2013), 12-17 May 2013, Shanghai, China, pp. 1874-1876. TUPWO001.

Loew, G. A. \& Altenmueller, O. H. (1965). Design and applications of $R F$ deflecting structures at SLAC. SLAC Report PUB-135. SLAC National Accelerator Laboratory, CA, USA.

Nagaoka, R. \& Wrulich, A. F. (2007). Nucl. Instrum. Methods Phys. Res. A, 575, 292-304.

Ratner, D. F. \& Chao, A. W. (2010). Phys. Rev. Lett. 105, 154801.

Shin, S., Kwon, S., Kim, D., Kim, D., Kim, M., Kim, S., Kim, S., Kim, J., Kim, C., Park, B., Park, S., Park, S., Park, E., Son, Y., Yoon, J., Lee, B., Lee, E., Lee, J., Lee, H., Joo, Y., Choi, J., Ha, T., Hwang, W., Hwang, I., Lee, J., Oh, B., Lee, C., Lee, H., Kim, J., Hwang, J. Y., Nam, S. H. \& Cho, M. (2013). J. Instrum. 8, P01019.

Steier, C., Byrd, J. M., De Santis, S., Nishimura, H., Robin, D., Sannibale, F., Sun, C., Venturini, M. \& Wan, W. (2016). Proceedings of the 7th International Particle Accelerator Conference (IPAC 2016), 8-13 May 2016, Busan, Korea, p. 2956.

Streun, A. (2014). Nucl. Instrum. Methods Phys. Res. A, 737, 148-154. Streun, A. (2017). Editor. SLS-2 Conceptual Design Report. Paul Scherrer Institut, Villigen, Switzerland.

Streun, A. \& Wrulich, A. (2015). Nucl. Instrum. Methods Phys. Res. $A, \mathbf{7 7 0}, 98-112$.

Xiang, D. \& Ding, Y. (2010). Phys. Rev. ST Accel. Beams, 13, 094001. Zholents, A. (2015). Nucl. Instrum. Methods Phys. Res. A, 798, 111116. 